\title{
Optical reference frame defined by Carlsberg Meridian Catalogue La Palma Number 4
}

LV Morrison \& RW Argyle, Royal Greenwich Observatory, UK

L Helmer, C Fabricius \& OH Einicke, Copenhagen University Observatory, DK

L Quijano \& JL Muiños, Real Instituto y Observatorio de la Armada en

San Fernando, SP

\begin{abstract}
The contribution of the Carlsberg Meridian Catalogue La Palma Number 4 to the determination of the optical reference frame is discussed. This catalogue of almost 51000 stars provides one of the most accurate optical reference frames at the present epoch, having a density of 1 star per square degree, and an average accuracy of 0.12 in position and $0 " 003$ per year in proper motion for stars with $\mathrm{V}<9$. The catalogue also contains positions of reference stars with $\mathrm{V}>11$ in the fields of benchmark extragalactic radio sources which can be used in linking the optical reference frame defined by the FK5 to the extragalactic frame.
\end{abstract}

\section{Carlsberg Automatic Meridian Circle}

The Carlsberg Automatic Meridian Circle (CAMC) is operated jointly by Copenhagen University Observatory, the Royal Greenwich Observatory and the Real Instituto y Observatorio de la Armada en San Fernando at the Spanish Observatorio del Roque de los Muchachos of the Instituto de Astrofisica de Canarias situated on the island of La Palma. The astronomical coordinates are: $17^{\circ} 53^{\prime} 07^{\prime \prime} 8$ West, $\quad 28^{\circ} 45^{\prime} 52^{\prime \prime} 4$ North, $\quad 2326 \mathrm{~m}$ altitude.

The telescope has an aperture of $18 \mathrm{~cm}$. It has been in operation continuously since May 1984, except for short periods due to technical problems and maintenance.

The same moving-slit micrometer has been used throughout the period of observation of this catalogue, and the photoelectric circle-reading system has also remained unchanged. All the processes, from setting the telescope to the scanning of the star during meridian passage and the reading of the circle, were carried out completely automatically under the control of two minicomputers. A general description of the system is given by Helmer \& Morrison (1985).

The major changes to the instrumental system during the observation of the catalogue were as follows. An air circulation system was installed in the tube of the 
telescope in August 1985 in order to remove the internal refraction caused by the thermal stratification of the air (Høg \& Fabricius 1988). An azimuth mark $130 \mathrm{~m}$ to the south was observed from January 1986 onwards, and another $50 \mathrm{~m}$ to the north from December 1986. These were automatically observed hourly together with the line of collimation, the horizontal flexure, and the level and zenith point of the circle which were obtained by observing the reflected image in a nadir pool of mercury.

\section{Observational programme}

The observing list consisted of the following programmes (limited to declinations above $-45^{\circ}$, apart from the FK5 which was limited to $-55^{\circ}$ ):

TABLE 1. Carlsberg Meridian Catalogue No.4: observational programmes

\section{Reference frame programmes}

FK5

International Reference Stars

General Catalogue

Reference stars in radio fields

PZT stars

Reference stars in selected Schmidt fields

Geodetic stars $(\operatorname{Dec}+7$ to +10$)$

Radio stars

Hipparcos non-astrometric (Dec 0 to -25 )

Hipparcos poor SAO positions

Gliese catalogue of nearby stars

\section{Proper motion programmes}

F-type stars within 100pc

G-type $d \& g$ and K-type $g$ within 300pc

Nearby O-B associations

Sample of A5-G0 stars in NGP

Solar system

Mars

Callisto

Saturn

Titan

Uranus

Neptune

Minor planets (62)
Total

size

1500

32524

24297

11397

1957

1014

374

313

3708

1352

1114

10550

9243

5629

3098
No. stars

CMC No.4

21328

8957

6829

797

822

356

203

1687

1043

412

9209

5665

2548

399

No. obs.

CMC No.4

137

92

131

70

284

294

6940 


\section{Real-time selection}

The selection of celestial and calibration objects was performed in real-time according to a pre-assigned priority. About $7 \%$ of the time was spent on calibration objects, $73 \%$ on programme stars, $18 \%$ on FK5 stars and $2 \%$ on planets. The scanning time was a function of the magnitudes of the objects: stars brighter than 9.5 took $16 \mathrm{~s}$, those between 9.5 and 11.0 took 32s, and those fainter than 11.0 took $64 \mathrm{~s}$. FK5 stars at zenith distances greater than $60^{\circ}$ were observed for $32 \mathrm{~s}$, and those greater than $75^{\circ}$ for $64 \mathrm{~s}$, irrespective of their magnitude. In order to improve the efficiency of the observing, stars were observed up to 40 s from the meridian after November 1985.

The same observing slits $\left(33^{\prime \prime} \times 4^{\prime \prime}\right)$ were used for all objects except for Mars and Saturn which were observed in a pair of large slits $\left(83^{\prime \prime} \times 4^{\prime \prime}\right)$. Very long slits $\left(2^{\prime} \times 4^{\prime \prime}\right)$ were used in a special search mode to locate stars with very poor input positions. Observations of these stars were, however, carried out in the normal slits after resetting the telescope.

\section{Differential reduction to FK5}

The real-time reduction to micrometer coordinates of the photon counts was carried out as described in Helmer et al.(1984) and Helmer \& Morrison (1985). A differential reduction for each night was carried out the following day by the observer using interactive computer programs. When available and reliable, the hourly values of the instrumental calibrations were subtracted from the FK5 residuals before the least-squares adjustment was made for the whole night, which comprised about 9 FK5 stars per hour down to a zenith distance of $84^{\circ}$. Stars above declination $+67^{\circ}$ were observed at either upper or lower culmination. Effectively, on most nights the least-squares adjustment amounted to a single rotation for the whole night in right ascension and declination to bring the instrumental system into agreement with the average position of the FK5 stars. The resulting positions in the catalogue are therefore not subject to possible zonal systematic errors in the FK5 frame.

The atmospheric extinction and instrumental absorption was determined nightly by least-squares adjustment to about 50 photoelectric standards spread through the night.

The least-squares adjustment in position and magnitude was then applied to the programme stars and solar system objects. The positions and magnitudes were compiled daily into a catalogue, and stars with the requisite number of observations (normally 6) were automatically deleted from the observing list.

\section{Formation of the catalogue}

The scanning slit micrometer was replaced by a new one in March 1988; so it was decided to combine all the observations made in the period May 1984 to February 1988 into one catalogue, superseding the three annual catalogues published for 
1984,1985 and 1986. In total, observations carried out in 8872 hours of operation on 955 nights were compiled into this catalogue. This represents $68 \%$ efficiency in observing.

Before forming the unweighted means of the positions of programme stars, about $6 \%$ were rejected because their stellar images were badly defined, being either too faint or not properly centered in the slits. A further $1 \%$ were rejected because they had large residuals. One night was completely rejected in declination due to problems with the circle-reading system. In magnitude, 111 nights of observations were rejected because their photometric quality was too poor.

\section{Accuracy of the catalogue}

The internal standard deviation (mean error) of a single observation was calculated from the scatter of the observations about their means. These were averaged in $5^{\circ}$ ranges of declination and the resultant values for stars with $\mathrm{V}<10$ are plotted in Figure 1(a). The average error curves in right ascension, declination and magnitude are:

$\sigma_{\alpha}=0.187 \sec ^{0.6} z \quad \sigma_{\delta}=0 " 181 \sec ^{0.9} z \quad \sigma_{m}=0^{m} .055 \sec z$,

where $z$ is the zenith distance. The mean error as a function of magnitude, reduced to the zenith, is plotted in Figure 1(b). It follows from this Figure that the average curves in right ascension and declination are only valid for stars with $\mathrm{V}<11$, and the average curve in magnitude for stars with $\mathrm{V}<9$.

The standard error of a catalogue position (formed from the mean of $n$ single observations) has been investigated using stars observed on both clamps, assuming that the standard error follows the relation;

$\epsilon_{n}=\sqrt{a^{2}+\sigma^{2} / n}$,

where $a$ is the asymptotic mean error and $\sigma$ is the standard deviation. Evaluating this for $a=0 " 068$ in right ascension and $a=0 " 076$ in declination we derive the values in Table 2.

TABLE 2. Standard error of a catalogue position

\begin{tabular}{|c|c|c|c|c|c|c|c|c|}
\hline \multirow[b]{2}{*}{$\mathbf{n}$} & \multicolumn{2}{|c|}{$\begin{array}{c}\mathrm{z}=0^{\circ} \\
\delta=+29^{\circ}\end{array}$} & \multicolumn{2}{|c|}{$\begin{array}{c}\mathrm{z}=30^{\circ} \\
\delta=-1^{\circ},+59^{\circ}\end{array}$} & \multicolumn{2}{|c|}{$\begin{array}{c}\mathrm{z}=60^{\circ} \\
\delta=-31^{\circ},+89^{\circ}\end{array}$} & \multicolumn{2}{|c|}{$\begin{array}{l}\mathrm{z}=73.8^{\circ} \\
\delta=-45^{\circ}\end{array}$} \\
\hline & $\epsilon_{\alpha}$ & $\epsilon_{\delta}$ & $\epsilon_{\alpha}$ & $\epsilon_{\delta}$ & $\epsilon_{\alpha}$ & $\epsilon_{\delta}$ & $\epsilon_{\alpha}$ & $\epsilon_{\delta}$ \\
\hline 3 & ".13 & ".13 & ".14 & ".14 & ".18 & ".21 & ".24 & ".34 \\
\hline 4 & "12 & ".12 & ".12 & ".13 & ".16 & "19 & ".21 & ".30 \\
\hline 5 & ".11 & ".11 & ".11 & ".12 & ".14 & ".17 & "19 & ".27 \\
\hline 6 & ".10 & ".11 & ".11 & ".11 & ".13 & ".16 & 18. & ".25 \\
\hline 7 & 10 & ".10 & ".10 & ".11 & ".13 & ".15 & ".17 & ".23 \\
\hline
\end{tabular}



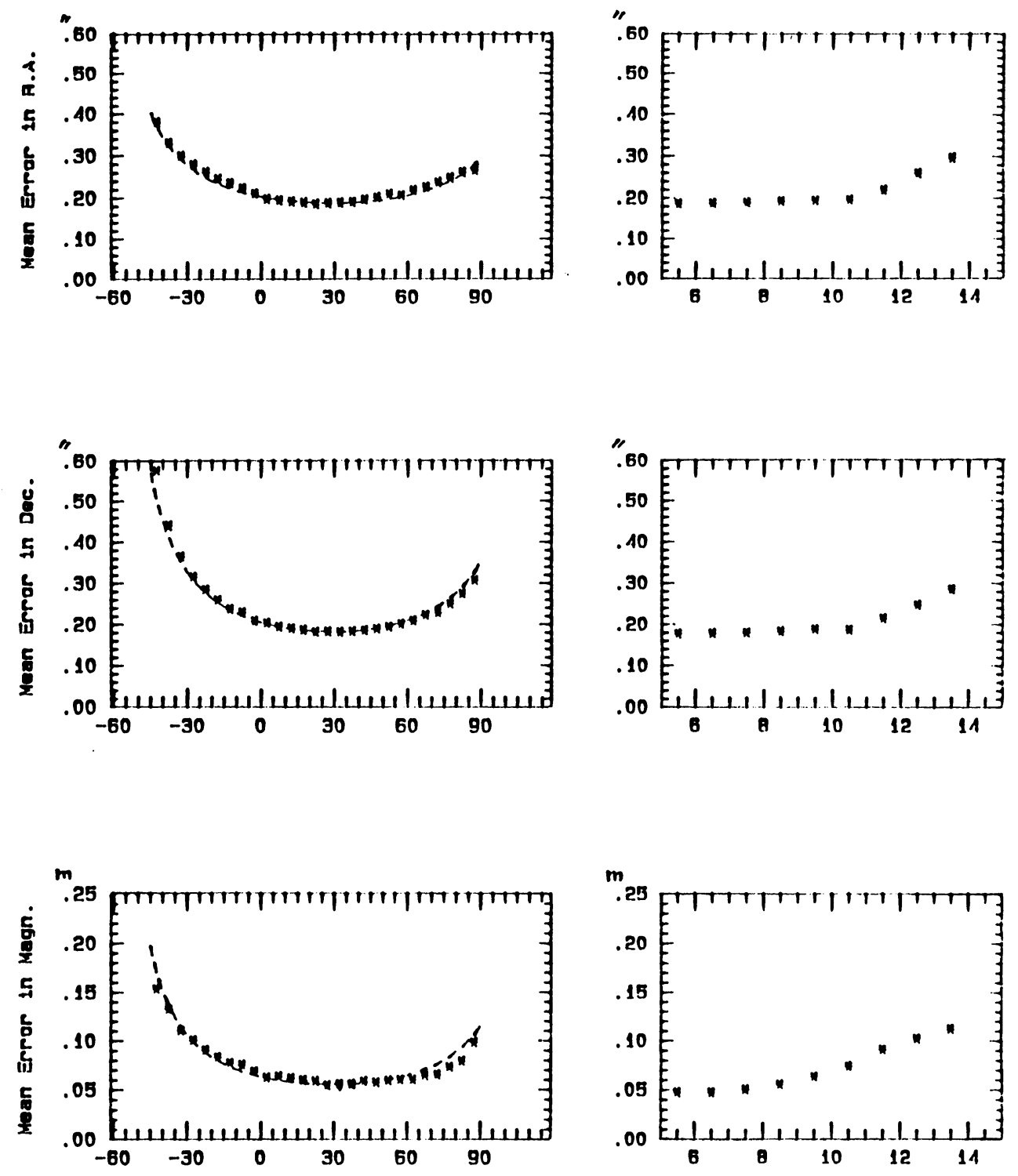

(a) Declination

(b) Magnitude

Figure 1. Mean error of a single observation as a function of (a) declination, and (b) magnitude. 


\section{Proper motions}

Proper motions were derived for almost 42000 of the stars by combining the position from this catalogue with positions from earlier epochs, using mainly the large photographic surveys of Yale, AGK2, AGK3 and Cape. Systematic corrections were applied to bring them on to the FK5. The distribution of the standard errors of the proper motions is shown in Figure 2. About 80\% are better than 0"004 per year.

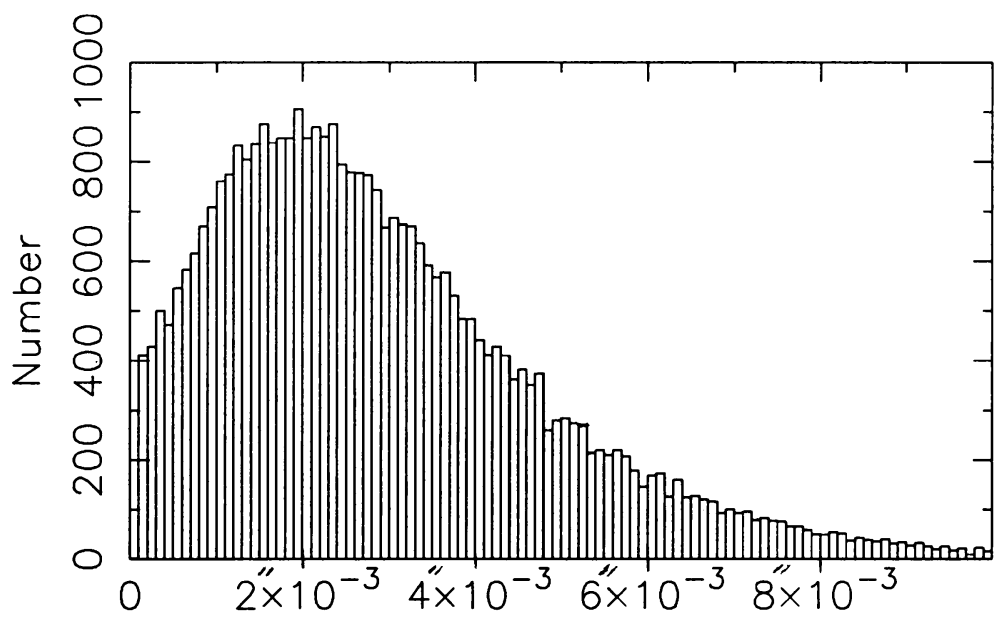

Figure 2. Distribution of standard errors of proper motions.

\section{Reference frame defined by this catalogue}

Figure 3 shows the distribution on the sky of the stars in this catalogue. Of the 51000 stars, about 34000 have $\mathrm{V}<9$ and these are evenly spread over the sky. About 21000 of these stars are IRS, the other 13000 coming from the first two proper motion programmes in Table 1. The square clumpy regions are selected Schmidt fields, whilst the irregular clumpy areas are reference stars in the fields of extragalactic radio sources. The trail of stars running down below the equator near $22^{\mathrm{h}}$ are the reference stars along the orbit of comet Halley which were observed in connection with the Giotto mission.

\subsection{An IRS-type reference frame}

The 34000 stars brighter than 9th magnitude should constitute a useful reference frame for photographic astrometry. The density is slightly greater than 1 star per square degree, which is comparable to the IRS; but, of course, it lacks the homogenity of spectral types, as there is a concentration towards F, G and K-types originating from the first two proper motion programmes of Table 1. Also, the streakiness in Figure 3 shows that there are a few areas, particularly around $7^{\mathrm{h}}$ and $8^{\mathrm{h}}$, where the density is less than 1 star per square degree. 


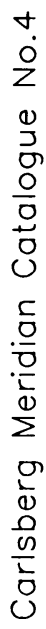

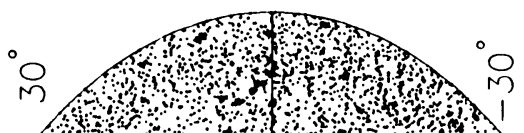

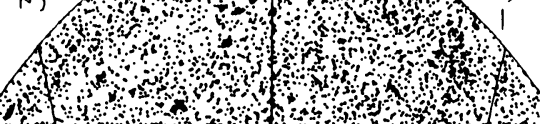

of

in

$\rightarrow+10$ ond

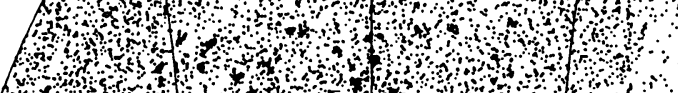

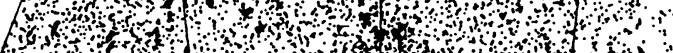

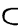

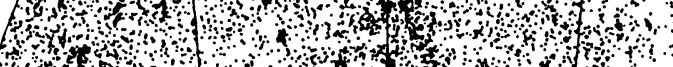

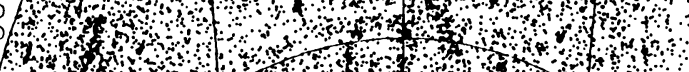

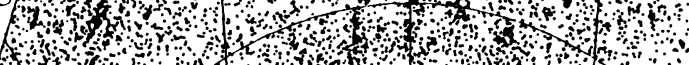

wom on

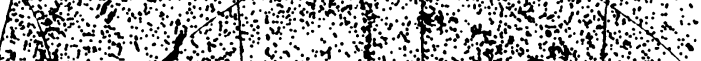

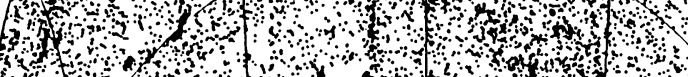

Hof

Fom

(f)

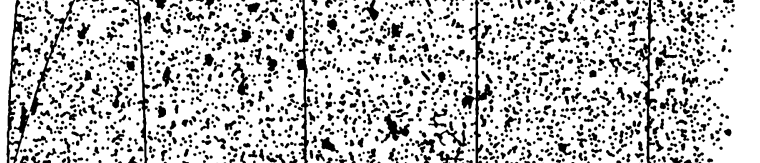

(3)

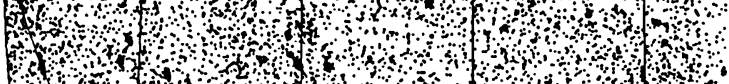

का

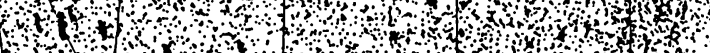

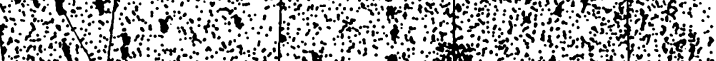

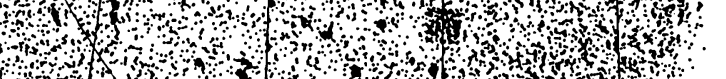

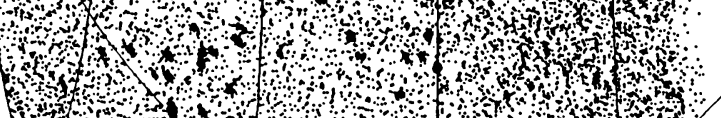

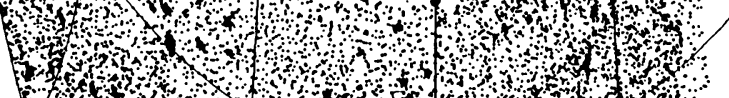

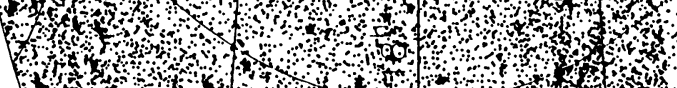

(1)

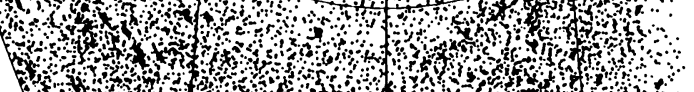

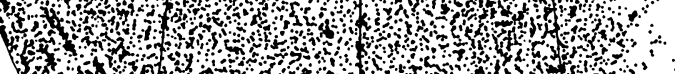

s.

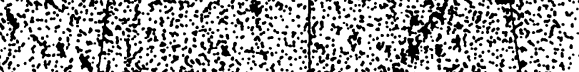

on

$0 \mathrm{fm}$ mom of

y

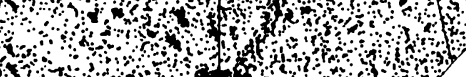

and

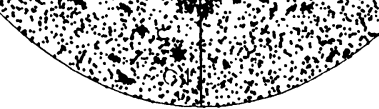




\subsection{Link to the extragalactic frame via quasars}

As can be seen from Table 1, the programme of observation of reference stars in the fields of extragalactic radio sources, mainly with $\mathrm{V}>11$, is slightly more than half way towards completion. Many fields do not yet have enough observed stars to reduce photographic plates satisfactorily. However, some fields that were given higher priority have been nearly completed, and work is proceeding in taking and measuring plates of these fields.

\subsection{Link to the extragalactic frame via radio stars}

Observations of radio stars have been carried out, and these have been combined with observations from the Bordeaux meridian circle in a comparative study with VLBI and VLA radio positions. This work is reported elsewhere in these conference proceedings (Morrison et al.).

\subsection{Reliability of the reference frame defined by this catalogue}

Comparisons have been carried out with the FK5 in order to check how closely the frame defined by this catalogue matches that of the FK5. Similar work was also carried out at Bordeaux, and a cross-comparison of the CAMC and Bordeaux frames (Morrison et al.1987) showed very good agreement over the the declination range of the comparison which extended from $-20^{\circ}$ to $+80^{\circ}$. The systematic differences, $\Delta \alpha_{\delta} \cos \delta$ and $\Delta \delta_{\delta}$, are reproduced here in Figure 4. The reference frames defined by CAMC and Bordeaux exhibit mutual agreement at the level of 0.03 , and indicate warps in the FK5 frame reaching $0{ }^{\prime \prime} 1$ in places at the epoch of the comparisons, 1986. Below $-30^{\circ}$, the CAMC frame is not so well tied down, particularly in declination. However, above $+80^{\circ}$ more investigations have been carried out in right ascension by combining observations at upper and lower culmination.

Figure 5 shows the differences $\Delta \alpha_{\delta} \cos \delta$ for CAMC-FK5 in the declination range $+68^{\circ}$ to $+90^{\circ}$. There is clear evidence of a systematic difference of $0 " 1$ around $+80^{\circ}$ to $+85^{\circ}$. Since the CAMC observations at both culminations, and thus at varying zenith distance, are in agreement, this suggests that most of the distortion lies in the FK5.

\section{Future programmes}

A new scanning slit micrometer was fitted to the telescope in March 1988, and came into full use in May 1988. This micrometer has improved sensitivity and accuracy, as reported in these proceedings by Fabricius et al. It is possible to reach $\mathrm{V}=14.5$ on a routine basis.

The completion of the IRS (to $\delta=-45^{\circ}$ ) and the reference stars in the fields of extragalactic radio sources are two of the main priorities of the present programme. 

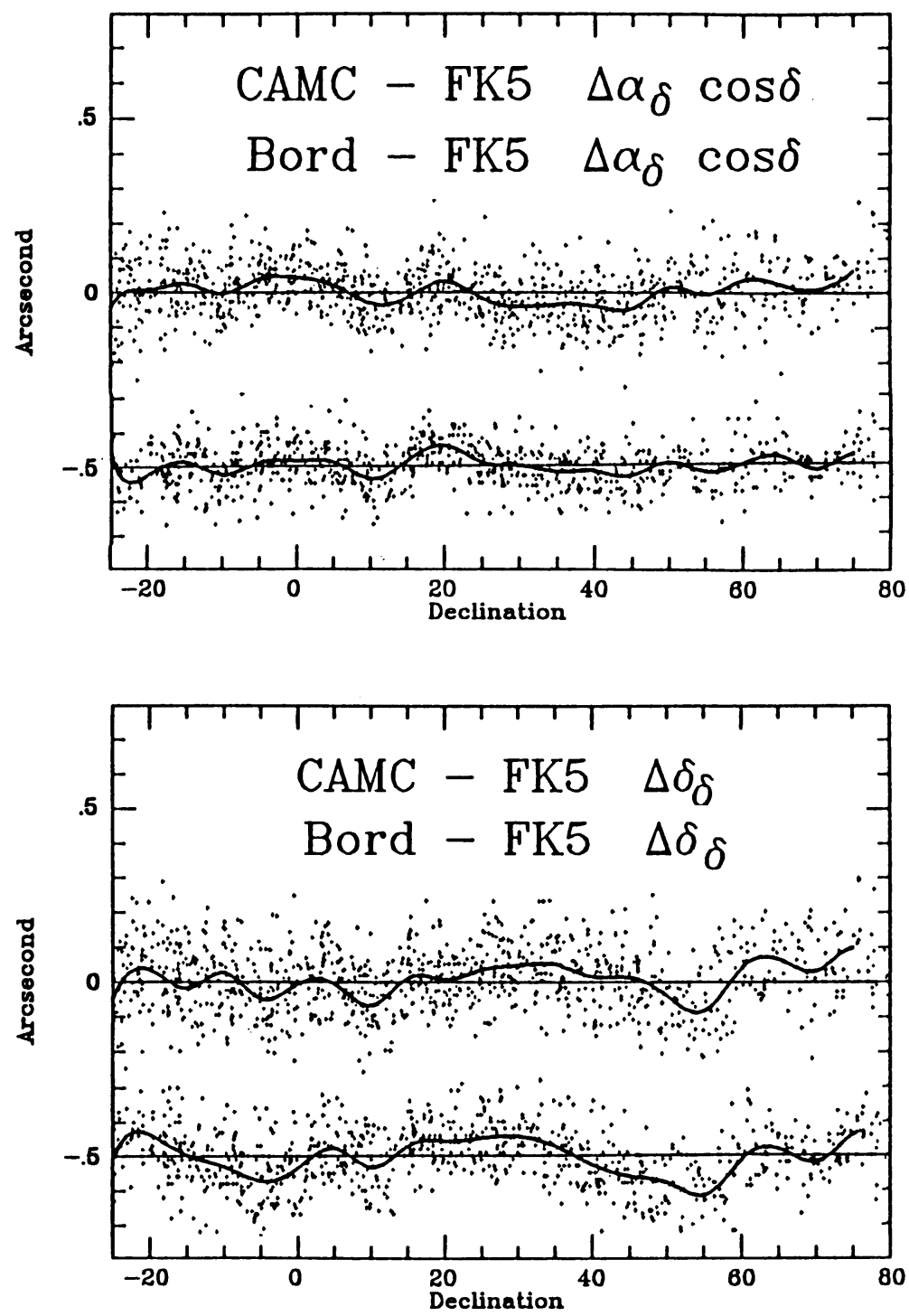

Figure 4. Systematic differences in right ascension and declination as a function of declination between CAMC and Bordeaux and FK5. The Bordeaux differences have been displaced by 0.5 in order to make the comparison clearer. 


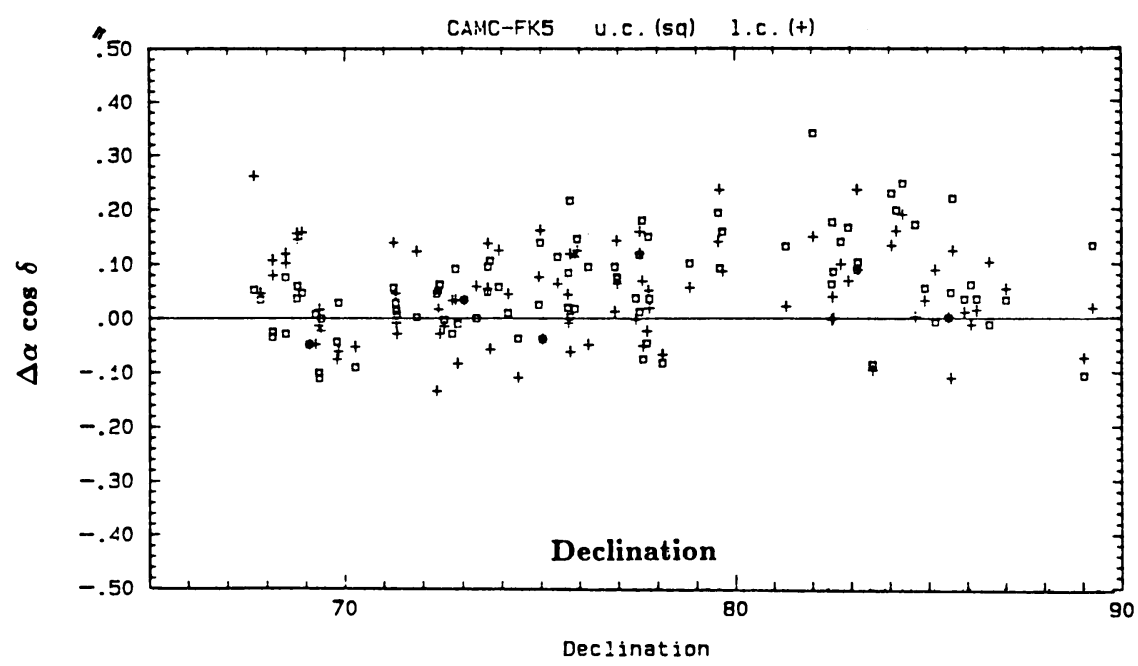

Figure 5. Differences in right ascension as a function of declination between CAMC and FK5 for stars observed at upper and lower culmination.

A new programme which has been started is the faint reference extension to the IRS. Stars have been selected with magnitude $V=11-12$ at a density of 1 star per square degree from the Astrographic Catalogue. So far, about 21000 stars have been included in the observing programme between declinations $-17^{\circ}$ to $+90^{\circ}$. Work is proceeding in selecting stars south of $-17^{\circ}$.

\section{References}

Fabricius, C., Helmer, L., Einicke, O.H., Morrison, L.V., Buontempo, M.E., Argyle, R.W., Quijano, L. and Muiños, J.L. (1990) 'First results from the new micrometer on the Carlsberg Automatic Meridian Circle', these proceedings.

Helmer, L., Fabricius, C., Einicke, O.H., Thoburn, C. and Morrison, L.V. (1984) 'Meridian observations made with the Carlsberg Automatic Meridian Circle at Brorfelde (Copenhagen University Observatory) 1982-1983, Astron. Astrophys. Suppl. Ser., 55, 87-102.

Helmer, L. and Morrison, L.V. (1985) 'Carlsberg Automatic Meridian Circle', Vistas in Astronomy, 28, 505-518.

$\mathrm{H} \phi \mathrm{g}$, E. and Fabricius, C. (1988) 'Atmospheric and internal refraction in meridian observations', Astron. Astrophys., 196, 301-312.

Morrison, L.V., Gibbs, P., Helmer, L., Fabricius, C., Einicke, O.H., Requième, Y. and Rapaport, M. (1990), 'Evidence of systematic errors in FK5', in IAU Coll. No.100 'Fundamentals of Astrometry' [Ed. Eichhorn], Belgrade, 1987. Kluwer Acad. Publ., Dordrecht.

Morrison, L.V., Argyle, R.W., Requième, Y. and Mazurier, J.M. (1990) 'Comparison of optical and radio positions of stars', submitted to Astron. Astrophys. 


\section{Discussion}

Mryamoто: Concerning the proper motion accuracy to be attained by Carlsberg Meridian Catalogues, could you tell me what kind of data for the first epoch observations will be used? MoRRISON: I have used mainly the large photographic catalogues: Yale, AGK2 and AGK3.

Pulyaev: How many polarissimae stars do you observe every night?

MORRISON: We observe 3 polar stars per hour, so it's approximately 30 per night.

HUGHES: What criterion or criteria do you use for rejecting observations? The genesis of my question is that $7 \%$ seems to be a high rejection rate and one might assume that some of the rejects are actually within a reasonable distribution.

MORRISON: The rejection figure of $7 \%$ largely comprises unsuccessful observations, such as those that were not properly scanned because of poor input positions. About $1 \%$ are rejected from criteria based on the size of the residual.

NEMIRO: Are there plans to use the Carlsberg instrument for determination of absolute positions? If not, it's a pity.

MORRISON: We do carry out observations which can eventually be used in an absolute discussion of the observations. For example, we observe three polarissimae each hour which can be used to define the absolute azimuth. 